\title{
AFFINE COMPLETE ORTHOLATTICES
}

\author{
DIETMAR SCHWEIGERT
}

ABstract. Every finite orthomodular lattice is affine complete.

In universal algebra the concept of the polynomial ring has been generalized to the concept of polynomial algebra $A\left[x_{1}, \ldots, x_{n}\right]$ of a given algebra $A$ in a variety [4]. By every polynomial $\psi\left(x_{1}, \ldots, x_{n}\right) \in A\left[x_{1}, \ldots, x_{n}\right]$ one can define a function $\psi: A^{n} \rightarrow A$ such that every element $\left(a_{1}, \ldots, a_{n}\right) \in A^{n}$ is mapped to $\psi\left(a_{1}, \ldots, a_{n}\right)$. These functions are called polynomial functions of $A$. Similarly, as in the variety of rings, the question of which functions $f$ : $A^{n} \rightarrow A$ are polynomial functions of $A$ is of major interest.

The function $f: A^{n} \rightarrow A$ is called compatible if for every congruence relation $\theta$ on the algebra $A, a_{1} \theta b_{1}, \ldots, a_{n} \theta b_{n}, a_{1}, \ldots, a_{n}, b_{1}, \ldots, b_{n} \in A$ imply $f\left(a_{1}, \ldots, a_{n}\right) \theta f\left(a_{1}, \ldots, a_{n}\right)$. Let us notice that every polynomial function is compatible [4]. The algebra $A$ is called polynomially complete if every function of $A$ is a polynomial function of $A$. In this case $A$ is simple and finite [4]. The algebra $A$ is called affine complete if every compatible function is a polynomial function of $A$ [6], [8]. A lot of examples can be found in [8] where it is also mentioned that finite Boolean algebras are affine complete. This result is essentially due to Grätzer [3] and it is our purpose to extend it to orthomodular lattices. In order to study polynomial functions on ortholattices we shall do it within a more general concept.

Definition. The algebra $\mathcal{L}=\left(L ; \wedge, \vee,{ }^{\prime}\right)$ is called a polarity lattice if $\mathcal{L}$ is a lattice concerning the operations $\wedge, \vee$ and if the unary operation ' has the properties

(1) $\left(x^{\prime}\right)^{`}=x$,

(2) $(x \vee y)^{\prime}=x^{\prime} \wedge y^{\prime}$.

It is clear that every ortholattice and every Boolean algebra are polarity lattices. We also have to use some results on order-polynomial-complete lattices [7]. The lattice $V$ is order-polynomial-complete if every order preserving function is a polynomial function of $V$. We call the polarity lattice $\mathcal{L}=\left(L ; \wedge, \vee,{ }^{\prime}\right)$ order-polynomial-complete if the underlying lattice $V=$ $(L ; \wedge, \vee)$ is order-polynomial-complete.

LEMMA. If the finite polarity lattice $L$ is order-polynomial-complete then $L$ is polynomially complete.

Received by the editors November 16, 1976.

AMS (MOS) subject classifications (1970). Primary 06A40.

(1) American Mathematical Society 1978 
Proof. Let $\{0, \ldots, a, \ldots, 1\}$ be the set of elements of $L$ and let us consider $f: L \rightarrow L$ with the decomposition of $f$ by

$$
f(x)=f_{0}(x) \vee \cdots \vee f_{a}(x) \vee \cdots \vee f_{1}(x)
$$

where

$$
f_{a}(x)= \begin{cases}f(a) & \text { if } x=a \\ 0 & \text { otherwise }\end{cases}
$$

For a further decomposition consider

$$
f_{a}(x)=\psi_{a}(x) \wedge \rho_{a}(x)
$$

where

$$
\psi_{a}(x)=\left\{\begin{array}{ll}
f(a) & \text { if } x \geqslant a, \\
0 & \text { otherwise, }
\end{array} \text { and } \rho_{a}(x)= \begin{cases}f(a) & \text { if } x \leqslant a, \\
0 & \text { otherwise. }\end{cases}\right.
$$

It is easy to see that $\psi_{a}$ is an order preserving function. By hypothesis $\psi_{a}$ is a polynomial function. Now consider

$$
\eta_{a}(x)= \begin{cases}f(a) & \text { if } x \geqslant a \\ 0 & \text { otherwise, }\end{cases}
$$

which is also order preserving and therefore a polynomial function. If we substitute $x^{`}$ for the variable $x$ in the polynomial function $\eta_{a}$, we get a polynomial function of the polarity lattice $L$ with $\eta_{a}\left(x^{\prime}\right)=\rho_{a}(x)$. Therefore $f$ is a polynomial function of $L$. If the unary functions of $L$ are polynomial functions, then the binary functions are polynomial functions, too. Consider $h: L^{2} \rightarrow L$ and decompose

$$
h(x, y)=h_{00}(x, y) \vee \cdots \vee h_{a b}(x, y) \vee \cdots \vee h_{11}(x, y),
$$

where

$$
h_{a b}(x, y)= \begin{cases}h(a, b) & \text { if } x=a \text { and } y=b, \\ 0 & \text { otherwise. }\end{cases}
$$

We have the further decomposition $h_{a b}(x, y)=\psi_{a b}(x) \wedge \rho_{a b}(y)$ where

$$
\psi_{a b}(x)=\left\{\begin{array}{ll}
f(a, b) & \text { if } x=a, \\
0 & \text { otherwise, }
\end{array} \text { and } \rho_{a b}(y)= \begin{cases}f(a, b) & \text { if } y=b, \\
0 & \text { otherwise. }\end{cases}\right.
$$

By Theorem 11.2 in [4] $L$ is polynomially complete.

THEOREM 1. The finite simple orthomodular lattice $L$ is polynomially complete.

Proof. It is well known that $L$ is relatively complemented [1, p. 53]. As $L$ is finite, Corollary 2 of $[1$, p. 53] implies that every element of $L \backslash\{0\}$ is the finite join of atoms of $L$. Therefore $L$ is atomistic and relatively complemented. The hypothesis for Theorem 10.14 of $[5$, p. 47] is fulfilled. As $L$ is simple it is also irreducible. By 10.14.3 of [5] the atoms of $L$ are projective to each other. That implies that the atoms of $L$ are pseudoprojective to each other in the sense of Definition 3 of [7]. Now by Satz 3 of [7] $L$ is 
order-polynomial-complete, and by the lemma $L$ is polynomially complete.

THEOREM 2. Every finite orthomodular lattice $L$ is affine complete.

Proof. If $L$ is a finite orthomodular lattice then $L$ is the direct product of simple orthomodular lattices which are polynomially complete. By [8, 4.9 Korollar] this is a diagonal product, and by [8, $5.6 \mathrm{Korollar}] L$ is affine complete.

\section{REFERENCES}

1. G. Birkhoff, Lattice theory, 3rd ed., Amer. Math. Soc. Colloq. Publ., vol. 25, Amer. Math. Soc., Providence, R.I., 1967.

2. G. Bruns and G. Kalmbach, Varieties or orthomodular lattices. II, Canad. J. Math. 24 (1972), 328-337.

3. G. Grätzer, On Boolean functions, Rev. Roumaine Math. Pures Appl. 7 (1962), 693-697.

4. H. Lausch and W. Nöbauer, Algebra of polynomials, Amsterdam, 1973.

5. F. Maeda and S. Maeda, Theory of symmetric lattices, Springer-Verlag, Berlin and New York, 1970.

6. A. F. Pixley, Completeness in arithmetical algebras, Algebra Universalis 2 (1972), 179-196.

7. D. Schweigert, Uber endliche, ordnungspolynomvollständige Verbände, Monatsh. Math. 78 (1974), 68-76.

8. H. Werner, Produkte von Kongruenzklassengeometrien universeller Algebren, Math. Z. 121 (1971), 111-140.

FB MAthematik, Universitït Kaiserslautern, 6750 Kaiserslautern, West Germany 